PROCEEDINGS OF THE

AMERICAN MATHEMATICAL SOCIETY

Volume 138, Number 8, August 2010, Pages 2641-2651

S 0002-9939(10)10320-7

Article electronically published on March 19, 2010

\title{
ON THE GLAUBERMAN CORRESPONDENT OF A BLOCK
}

\author{
YUANYANG ZHOU
}

(Communicated by Jonathan I. Hall)

\begin{abstract}
In this paper, we analyze the compatibility of Fong's reduction and the Glauberman correspondence of characters and then clarify that the $p$-solvable hypothesis in a paper of Harris and Linckelmann is not necessary.
\end{abstract}

Let $\mathcal{O}$ be a complete discrete valuation ring with an algebraically closed residue field $k$ of characteristic $p$ and a quotient field $\mathcal{K}$ of characteristic 0 . In addition, $\mathcal{K}$ is also assumed to be big enough for all finite groups that we consider below. Let $G$ be a finite group. We denote by $\operatorname{Irr}(G)$ the set of all irreducible characters of $G$. Let $A$ be another finite group acting on $G$. Then clearly $A$ acts on $\operatorname{Irr}(G)$. The following theorem is due to Glauberman 4 and relates the $A$-fixed $\operatorname{set}\left(\operatorname{Irr}_{\mathcal{K}}(G)\right)^{A}$ with the set $\operatorname{Irr}_{\mathcal{K}}\left(C_{G}(A)\right)$.

Theorem 1. For any A-group $G$ with $A$ solvable and $|G|$ and $|A|$ coprime, there is a bijection $\pi(G, A): \operatorname{Irr}_{\mathcal{K}}(G)^{A} \rightarrow \operatorname{Irr}_{\mathcal{K}}\left(C_{G}(A)\right)$ such that

1.1. For any normal subgroup $B$ of $A$, the bijection $\pi(G, B)$ maps $\left(\operatorname{Irr}_{\mathcal{K}}(G)\right)^{A}$ to $\left(\operatorname{Irr}_{\mathcal{K}}\left(C_{G}(B)\right)\right)^{A}$, and we have $\pi(G, A)=\pi\left(C_{G}(B), A / B\right) \circ \pi(G, B)$ on $\left(\operatorname{Irr}_{\mathcal{K}}(G)\right)^{A}$.

1.2. If $A$ is a $q$-group for some prime $q$, for any $\chi \in\left(\operatorname{Irr}_{\mathcal{K}}(G)\right)^{A}$ the corresponding irreducible character $\pi(G, A)(\chi)$ of $C_{G}(A)$ is the unique irreducible constituent of $\operatorname{Res}_{C_{G}(A)}^{G}(\chi)$ occurring with a multiplicity prime to $q$.

2. Continue to keep the notation in Theorem 1 . Let $b$ be a block idempotent of $\mathcal{O} G$. We denote by $\operatorname{Irr}(G, b)$ the set of all irreducible characters of $G$ provided by some $\mathcal{K} G b$-module. If $A$ centralizes a defect group $P$ of $b$, by 9 , Prop. 1 and Th. 1], $A$ stabilizes all characters of $\operatorname{Irr}(G, b)$ and there is a unique block idempotent $c$ of $\mathcal{O} C_{G}(A)$ such that $\operatorname{Irr}\left(C_{G}(A), c\right)=\pi(G, A)(\operatorname{Irr}(G, b))$; moreover $b$ and $c$ are perfectly isometric (refer to [1]). Such a block idempotent $c$ is called the Glauberman correspondent of $b$ (see [9]). A perfect isometry between blocks is considered to be the character-theoretic 'shadow' of a derived equivalence. So it is very interesting to ask whether there is such a derived equivalence between a block and its Glauberman correspondent. More strongly, with the $p$-solvable hypothesis, Harris and Linckelmann proved that a block and its Glauberman correspondent are Morita equivalent. In this paper, we analyze the compatibility of Fong's reduction and the Glauberman correspondence of characters and then clarify that the $p$-solvable hypothesis in [5] is not necessary.

Received by the editors May 14, 2009.

2010 Mathematics Subject Classification. Primary 20C15, 20C20.

Key words and phrases. Characters, blocks, the Glauberman correspondent.

(C)2010 American Mathematical Society

Reverts to public domain 28 years from publication 
Theorem 3. Let $G$ be an A-group with $A$ solvable and $|G|$ and $|A|$ be co-prime. Let $b$ be a block idempotent of $\mathcal{O} G$ with a defect group $P$ centralized by $A$ and let $c$ be the Glauberman correspondent of b (refer to [9]). If $G=O_{p^{\prime}}(G) C_{G}(A)$, then there is an indecomposable $\mathcal{O}\left(G \times C_{G}(A)\right)$-module $M$ having the following properties:

3.1. The $p$-subgroup $\Delta(P)=\{(x, x) \mid x \in P\}$ of $G \times C_{G}(A)$ is a vertex of $M$.

3.2. $M$ induces a Morita equivalence between $\mathcal{O} G b$ and $\mathcal{O} C_{G}(A) c$, and the bijection between $\operatorname{Irr}(G, b)$ and $\operatorname{Irr}\left(C_{G}(A), c\right)$ induced by this Morita equivalence coincides with the Glauberman correspondence from $\operatorname{Irr}(G, b)$ to $\operatorname{Irr}\left(C_{G}(A), c\right)$.

4. Firstly we introduce some notation and terminology used in this paper. Let $S$ be a set and $H$ be a group acting on $S$. For any $h \in H$ and $s \in S$, we write the action of $h$ on $s$ as $h \cdot s$. Let $A$ be an $\mathcal{O}$-algebra with the identity element. We denote by $A^{*}$ the multiplicative group of all invertible elements of $A$. Let $K$ be a finite group. A group $\hat{K}$ is an $\mathcal{O}^{*}$-group with the $\mathcal{O}^{*}$-quotient $K$ if there is an injective group homomorphism $\rho: \mathcal{O}^{*} \rightarrow Z(\hat{K})$ such that $\hat{K} / \rho\left(\mathcal{O}^{*}\right) \cong K$. Then $\hat{K}$ can be regarded as a central extension of $K$ by $\mathcal{O}$, and, in this case, we denote by $\mathcal{O}_{*} \hat{K}$ the twisted group algebra corresponding to this central extension (see [10, 10.4]). For any $\lambda \in \mathcal{O}^{*}$ and $x \in \hat{K}$, we write the product $\rho(\lambda) x$ as $\lambda \cdot x$ for convenience. For any subgroup $L$ of $K$, we denote by $\hat{L}$ the inverse image of $L$ through the canonical surjective homomorphism $\hat{K} \rightarrow K$; then $\hat{L}$ is an $\mathcal{O}^{*}$ group with the $\mathcal{O}^{*}$-quotient $L$. The group $\hat{K}$ with another injective homomorphism $\mathcal{O}^{*} \rightarrow Z(\hat{K}), \lambda \mapsto \rho\left(\lambda^{-1}\right)$ is also an $\mathcal{O}^{*}$-group with the $\mathcal{O}^{*}$-quotient $K$. We call this $\mathcal{O}^{*}$-group the opposite $\mathcal{O}^{*}$-group of $\hat{K}$ and denote it by $\hat{K}^{\circ}$. Let $\hat{H}$ be an $\mathcal{O}^{*}$-group with the $\mathcal{O}^{*}$-quotient $H$. A homomorphism $\theta: \hat{K} \rightarrow \hat{H}$ is called a homomorphism of $\mathcal{O}^{*}$-groups if for any $\lambda \in \mathcal{O}^{*}$ and $x \in \hat{K}$, we have $\theta(\lambda \cdot x)=\lambda \cdot \theta(x)$. Below, we will occasionally use the induction of interior $K$-algebras, the Brauer quotients and the Brauer homomorphisms; for their definitions and corresponding notation, readers can refer to [10; by the word "character", we sometimes indicate a character on a group and sometimes indicate a character on an algebra, but that does not cause any confusion.

Next we begin to analyze the compatibility of Fong's reduction and the Glauberman correspondence of characters. Let $G$ be a finite group, $b$ be a block idempotent of $\mathcal{O} G$ and $P$ be a defect group of $b$. Let $A$ be another finite group acting on $G$. We assume that $|G|$ and $|A|$ are coprime, $O_{p^{\prime}}(G) \cap C_{G}(A)=O_{p^{\prime}}\left(C_{G}(A)\right), A$ is cyclic with the order a power of some prime $q$ and $A$ centralizes $P$. Let $c$ be the Glauberman correspondent of $b$. Set $C=C_{G}(A)$ and let $R$ be a Sylow $p$-subgroup of $C$ containing $P$.

5. Let $I$ be the set of all block idempotents $f$ of $\mathcal{O} O_{p^{\prime}}(G)$ such that $b f \neq 0$. The action of $A$ on $O_{p^{\prime}}(G)$ induces an action of $A$ on $\mathcal{O} O_{p^{\prime}}(G)$, which again induces an action of $A$ on $I$. We consider the action of the semidirect $G \rtimes A$ defined by the equality $g \cdot f=g f g^{-1}$, where $g \in G$ and $f \in I$, together with the action of $A$ on $I$. By this action, it is well known that $G$ acts transitively on $I$; moreover since $|G|$ and $|A|$ are co-prime, by [3, Lemma 13.8 and Cor. 13.9], the set $I^{A}$ of $A$-fixed points in $I$ is non-empty and $C_{G}(A)$ acts transitively on $I^{A}$. We denote by $K$ the stabilizer of $f$ in $G$ and set $\operatorname{Tr}_{K}^{G}(f)=\sum_{x \in G / K} x f x^{-1}$, where $G / K$ is a set of representatives of right cosets of $K$ in $G$. Clearly $f f^{x}=0$ for any $x \in G-K$; 
therefore by [3, 2.6.3], we have an isomorphism

$$
\operatorname{Ind}_{K}^{G}(\mathcal{O} K f) \cong \mathcal{O} G \operatorname{Tr}_{K}^{G}(f)
$$

which maps $1 \otimes y \otimes 1$ onto $y$ for any $y \in \mathcal{O} K f$.

6. Since any element of $Z\left(\operatorname{Ind}_{K}^{G}(\mathcal{O} K f)\right)$ can be written as the sum $\sum_{x \in G / K} x \otimes$ $a \otimes x^{-1}$ for some $a \in Z(\mathcal{O} K f)$, by the isomorphism (5.1), the map

$$
Z(\mathcal{O} K f) \rightarrow Z\left(\mathcal{O} G \operatorname{Tr}_{K}^{G}(f)\right), a \mapsto \sum_{x \in G / K} x a x^{-1}
$$

is an isomorphism. Obviously $Z(\mathcal{O} K f)$ and $Z\left(\mathcal{O} G \operatorname{Tr}_{K}^{G}(f)\right)$ are both $A$-stable and the isomorphism (6.1) is compatible with the $A$-actions on $Z(\mathcal{O} K f)$ and $Z\left(\mathcal{O} G \operatorname{Tr}_{K}^{G}(f)\right)$. Since $b \operatorname{Tr}_{K}^{G}(f)=b, b \in Z\left(\mathcal{O} G \operatorname{Tr}_{K}^{G}(f)\right)$ and we denote by $d$ the inverse image of $b$ through (6.1), which is $A$-stable. Then $b=\sum_{x \in G / K} x d x^{-1}$ and $d x d x^{-1}=0$ for any $x \in G-K$. Again by [3, 2.6.3], we have an isomorphism

$$
\operatorname{Ind}_{K}^{G}(\mathcal{O} K d) \cong \mathcal{O} G b
$$

mapping $1 \otimes y \otimes 1$ onto $y b$ for any $y \in \mathcal{O} K d$.

7. Let $J$ be the set of all defect groups of $d$. It is well known that $K$ acts transitively on $J$. Since $A$ stabilizes $d, A$ also acts on $J$. We consider the action of $K \rtimes A$ on $J$ defined by these two actions of $A$ and $K$ on $J$. Since $|A|$ and $|K|$ are co-prime, by [3. Lemma 13.8 and Cor. 13.9], the $J^{A}$ of all $A$-stable elements of $J$ is non-empty and $C_{K}(A)$ acts transitively on $J^{A}$. Similarly $b$ also has $A$-stable defect groups and $C_{G}(A)$ acts transitively on all these defect groups. By the isomorphism (6.2), all defect groups of $d$ are also defect groups of $b$. Since $P$ is a defect group centralized by $A$, adjusting $K$ and $d$ by suitable $C_{G}(A)$-conjugation, we can assume that $P \leq K$ and $P$ is a defect group of $d$. Let $e$ and $g$ be the Glauberman correspondents of $d$ and $f$ respectively. Since we assume $O_{p^{\prime}}(G) \cap C_{G}(A)=O_{p^{\prime}}\left(C_{G}(A)\right), g$ is actually a block idempotent of $\mathcal{O} O_{p^{\prime}}\left(C_{G}(A)\right)$.

Proposition 8. Keep the notation and hypotheses as above. Then the stabilizer of $g$ under the $C_{G}(A)$-conjugation is equal to $C_{K}(A)$, ge $=e$ and there is an isomorphism

$$
\operatorname{Ind}_{C_{K}(A)}^{C_{G}(A)}\left(\mathcal{O} C_{K}(A) e\right) \cong \mathcal{O} C_{G}(A) c .
$$

Proof. It is trivial to see that [5, Th. 5.1] still holds without the $p$-solvable condition.

Now we assume that the block idempotent $f$ of $\mathcal{O} O_{p^{\prime}}(G)$ is stabilized by $G \rtimes A$; then $K=G, d=b, e=c$ and $C_{K}(A)=C_{G}(A)$.

9. Obviously $G$ acts on the full matrix algebra $\mathcal{O} O_{p^{\prime}}(G) f$ over $\mathcal{O}$, and by the Skolem-Noether theorem, there exists a group homomorphism

$$
\rho: G \rightarrow \operatorname{Aut}\left(\mathcal{O} O_{p^{\prime}}(G) f\right) \cong\left(\mathcal{O} O_{p^{\prime}}(G) f\right)^{*} / \mathcal{O}^{*} .
$$

We denote by $\hat{G}$ the set of all elements $(x, s)$ such that $\rho(x)$ is the image of $s$ in $\left(\mathcal{O} O_{p^{\prime}}(G) f\right)^{*} / \mathcal{O}^{*}$, where $s \in\left(\mathcal{O} O_{p^{\prime}}(G) f\right)^{*}$ and $x \in G$. $\hat{G}$ is a group with respect to the multiplication $(x, s)\left(x^{\prime}, s^{\prime}\right)=\left(x x^{\prime}, s s^{\prime}\right)$. Endowed with the homomorphism $\mathcal{O}^{*} \rightarrow \hat{G}$ mapping $\lambda$ onto $(1, \lambda), \hat{G}$ becomes an $\mathcal{O}^{*}$-group with the $\mathcal{O}^{*}$-quotient $G$. There is an injective group homomorphism $O_{p^{\prime}}(G) \rightarrow \hat{G}, x \mapsto(x, x b)$, whose image is normal in $\hat{G}$ and intersects $\mathcal{O}^{*}$ trivially. Later we always identify $O_{p^{\prime}}(G)$ with this image through the injective homomorphism. 
10. We set

$$
\bar{G}=G / O_{p^{\prime}}(G) \quad \text { and } \quad \hat{\bar{G}}=\hat{G} / O_{p^{\prime}}(G) .
$$

For any $(x, s) \in \hat{G}$, we denote by $\overline{(x, s)}$ the image of $(x, s)$ in $\bar{G}$. With the obvious injective homomorphism $\mathcal{O}^{*} \rightarrow \hat{G} / O_{p^{\prime}}(G)$ mapping $\lambda$ onto $\overline{(1, \lambda)}, \hat{\bar{G}}$ is an $\mathcal{O}^{*}$-group with the $\mathcal{O}^{*}$-quotient $\bar{G}$. The actions of $A$ on $\mathcal{O} O_{p^{\prime}}(G) g$ and $G$ induce an action of $A$ on $\hat{G}$ defined by the equality

$$
a \cdot(x, s)=(a \cdot x, a \cdot s)
$$

for any $a \in A$ and $(x, s) \in \hat{G}$. Obviously $O_{p^{\prime}}(G)$ is an $A$-stable normal subgroup of $\hat{G}$ and thus the action of $A$ on $\hat{G}$ induces an action of $A$ on $\hat{\bar{G}}$. Set $m=$ $|G|\left|O_{p^{\prime}}(G)\right|$ and denote by $\mu_{m}$ the subgroup of $\mathcal{O}^{*}$ of all $m$-th roots of unity. For any $x \in G$, choose $s_{x} \in\left(\mathcal{O} O_{p^{\prime}}(G) f\right)^{*}$ such that $\left(x, s_{x}\right) \in \hat{G}$ and $\operatorname{det}\left(s_{x}\right)=1$. Set $G^{\prime}=\left\{\left(x, \lambda s_{x}\right) \mid \lambda \in \mu_{m}, x \in G\right\}$.

Lemma 11. Keep the notation and hypotheses as above. Then $G^{\prime}$ is an A-stable finite subgroup of $\hat{G}$ such that $\hat{G}=\mathcal{O}^{*} G^{\prime}$ and $O_{p^{\prime}}(G) \leq G^{\prime}$; moreover, the exponent of $G^{\prime}$ divides $m$.

Proof. This lemma easily follows from the proof of [5, 4.5 (i)].

12. Since $\mathcal{O} O_{p^{\prime}}(G) f$ is a full matrix algebra over $\mathcal{O}$ and its rank over $\mathcal{O}$ is prime to $p$, the restriction to $R$ of the homomorphism $\rho$ can be lifted to a unique homomorphism $\sigma: R \rightarrow\left(\mathcal{O} O_{p^{\prime}}(G) f\right)^{*}$ such that $\operatorname{det}(\sigma(u))=1$ for any $u \in R$ (refer to [7, para. 6.2]). By this homomorphism, we define a new homomorphism $R \rightarrow \hat{G}, u \mapsto(u, \sigma(u))$, which is injective and whose image intersects $\mathcal{O}^{*}$ trivially. We identity $R$ with its image through this homomorphism. We claim that

$$
R \subset C_{G^{\prime}}(A) .
$$

Firstly, since $\mathcal{O} O_{p^{\prime}}(G) f$ is a full matrix algebra over $\mathcal{O}$, by the Skolem-Noether theorem, for any $a \in A$ and $u \in R, a(\sigma(u))$ is equal to some conjugate of $\sigma(u)$ in $\mathcal{O} O_{p^{\prime}}(G) f$ and thus $\operatorname{det}(a(\sigma(u)))=1$; then it follows from the uniqueness of the homomorphism $\sigma$ that $a(\sigma(u))=\sigma(u)$ for any $a \in A$ and $u \in R$. This shows that $A$ centralizes the subgroup $R$ of $\hat{G}$. Secondly it is clear that $s_{u} \sigma(u)^{-1}$ is an invertible element of $\mathcal{O}$ with order dividing $m$. Therefore there is some $\lambda \in \mu_{m}$ such that $s_{u}=\lambda \sigma(u)$ and then $(u, \sigma(u)) \in G^{\prime}$. The claim is proved.

13. By [5, Th. 4.4], there is an algebra isomorphism

$$
\mathcal{O} G f \cong \mathcal{O} O_{p^{\prime}}(G) f \otimes_{\mathcal{O}} \mathcal{O}_{*} \hat{\bar{G}}^{\circ}
$$

mapping $x f$ onto $s \otimes \overline{(x, s)}$ for any $x \in G$. Since $f b=b$, this isomorphism determines a central primitive idempotent $\bar{b}$ of $\mathcal{O}_{*} \hat{\bar{G}}^{\circ}$ such that

$$
\mathcal{O} G b \cong \mathcal{O} O_{p^{\prime}}(G) f \otimes_{\mathcal{O}} \mathcal{O}_{*} \hat{\bar{G}}^{\circ} \bar{b} .
$$

Moreover the image of $P$ in $\bar{G}$ is a defect group of $\bar{b}$ (refer to [8, Cor. 6.6]). Since $P$ and the image of $P$ in $\bar{G}$ are isomorphic, we identify them. We let $\alpha$ be the unique character of $\mathcal{O} O_{p^{\prime}}(G) f$ and denote by $\operatorname{Irr}\left(\hat{\bar{G}}^{\circ}, \bar{b}\right)$ the set of characters of all 
irreducible $\mathcal{K} \otimes_{\mathcal{O}} \mathcal{O}_{*} \hat{\bar{G}}^{\circ} \bar{b}$-modules. Then by the isomorphism (13.2), we obtain a bijection

$$
\operatorname{Irr}(G, b) \rightarrow \operatorname{Irr}\left(\hat{\bar{G}}^{\circ}, \bar{b}\right), \chi \mapsto \bar{\chi}
$$

such that for any $x \in G, \chi(x)=\alpha(s) \bar{\chi}(x, s)$.

14. By paragraph 9 applied to $C, \mathcal{O} O_{p^{\prime}}(C)$ and $g$, we obtain a group homomorphism

$$
\varrho: C \rightarrow \operatorname{Aut}\left(\mathcal{O} O_{p^{\prime}}(C) g\right) \cong\left(\mathcal{O} O_{p^{\prime}}(C) g\right)^{*} / \mathcal{O}^{*} .
$$

By this homomorphism, we construct an $\mathcal{O}^{*}$-group $\tilde{C}$ with the $\mathcal{O}^{*}$-quotient $C$, which is the set of all elements $(y, t)$ such that $\varrho(y)$ is the image of $t$ in $\left(\mathcal{O} O_{p^{\prime}}(C) g\right)^{*} / \mathcal{O}^{*}$, where $t \in\left(\mathcal{O} O_{p^{\prime}}(C) g\right)^{*}$ and $y \in C$. Just as $\hat{G}$ in paragraph $9, \hat{C}$ has three subgroups: the first is the normal group $\left\{(y, y g) \mid y \in O_{p^{\prime}}(C)\right\}$ and we identity this normal subgroup with $O_{p^{\prime}}(C)$; the second is the subgroup $C^{\prime}$ consisting of all elements $\left(y, \lambda t_{y}\right)$, where $\lambda \in \mu_{m}, y \in C$ and $t_{y}$ belongs to $\left(\mathcal{O} O_{p^{\prime}}(C) g\right)^{*}$ such that $\left(y, t_{y}\right) \in \tilde{C}$ and $\operatorname{det}\left(t_{y}\right)=1$; the third is the $p$-group $R=\{(u, \varsigma(u)) \mid u \in R\}$, where $\varsigma: R \rightarrow\left(\mathcal{O O}_{p^{\prime}}(C) g\right)^{*}$ is the unique lifting of the restriction of $\varrho$ to $R$ such that $\operatorname{det}(\varsigma(u))=1$ for any $u \in R$. Note that the exponent of $C^{\prime}$ also divides $m$ (refer to Lemma 11) and that $R \subset C^{\prime}$ (refer to (12.1)). We set

$$
\bar{C}=C / O_{p^{\prime}}(C) \text { and } \tilde{\bar{C}}=\tilde{C} / O_{p^{\prime}}(C)
$$

and denote by $\overline{(x, t)}$ the image of $(x, t)$ in $\tilde{\bar{C}}$ for any $(x, t) \in \tilde{C}$. Then $\tilde{\bar{C}}$ is an $\mathcal{O}^{*}$-group with $\mathcal{O}^{*}$-quotient $\bar{C}$.

15. By [5, Th. 4.4], there is an algebra isomorphism

$$
\mathcal{O} C g \cong \mathcal{O} O_{p^{\prime}}(C) g \otimes_{\mathcal{O}} \mathcal{O}_{*} \tilde{\bar{C}}^{\circ}
$$

mapping $x g$ onto $t \otimes \overline{(x, t)}$ for any $x \in C$. Since $g c=c$, this isomorphism determines a central primitive idempotent $\bar{c}$ of $\mathcal{O}_{*} \tilde{\bar{C}}^{\circ}$ such that

$$
\mathcal{O} C c \cong \mathcal{O} O_{p^{\prime}}(C) g \otimes_{\mathcal{O}} \mathcal{O}_{*} \tilde{\bar{C}}^{\circ} \bar{c} .
$$

Let $\beta$ be the unique character of $\mathcal{O} O_{p^{\prime}}(C) g$ and denote by $\operatorname{Irr}\left(\tilde{\bar{C}}^{\circ}, \bar{c}\right)$ the set of characters of all irreducible $\mathcal{K} \otimes_{\mathcal{O}} \mathcal{O}_{*} \tilde{\bar{C}}^{\circ} \bar{c}$. By the isomorphism (15.2), we obtain a bijection

$$
\operatorname{Irr}(C, c) \rightarrow \operatorname{Irr}\left(\tilde{\bar{C}}^{\circ}, \bar{c}\right), \psi \mapsto \bar{\psi}
$$

such that for any $x \in C, \psi(x)=\beta(t) \bar{\psi}(x, t)$.

Lemma 16. Keep the notation and hypotheses as above. Then $C_{\hat{G}}(A)=\hat{C}$ and $C_{G^{\prime}}(A)$ is a subgroup of $\hat{C}$ such that $\mathcal{O}^{*} C_{G^{\prime}}(A)=\hat{C}$.

Proof. Take $x \in C$ and let $K$ be the set of all the inverse images of $x$ through the canonical surjective homomorphism $G^{\prime} \rightarrow G$. Then since it follows from Lemma 11 that $G^{\prime}$ is $A$-stable, $A$ stabilizes $K$. Consider the action of $\mu_{m} \times A$ on $K$ defined by the left multiplication of $\mu_{m}$ on $K$ and the above $A$-action on $K$ together. Since $\mu_{m}$ acts regularly on $K$ and $A$ and $\mu_{m}$ have co-prime order, by [3, Lemma 13.8 and Cor. 13.9], $A$ has to stabilize some element of $K$ and $\mu_{m}$ regularly acts on these $A$-stabilized elements; in particular, $A$ stabilizes all elements of $K$. This shows that $C_{G^{\prime}}(A)$ is a subgroup of $\hat{C}$ such that $\mathcal{O}^{*} C_{G^{\prime}}(A)=\hat{C}$. Thus $C_{\hat{G}}(A)=\hat{C}$. 
The following two lemmas will be used to analyze the local structure of the $\mathcal{O}\left(G \times C_{G}(A)\right)$-module in Theorem 3 inducing Morita equivalence between $\mathcal{O} G b$ and $\mathcal{O} C_{G}(A) c$. For the compatibility of Fong's reduction with the Glauberman correspondence of characters (see Proposition 23 below), they can be skipped for now.

Lemma 17. Keep the notation and hypotheses as above. There is an isomorphism

$$
\Phi: C_{G^{\prime}}(A) \cong C^{\prime},
$$

which preserves $R$ and $O_{p^{\prime}}(C)$ element-wise and makes the following diagram commutative:

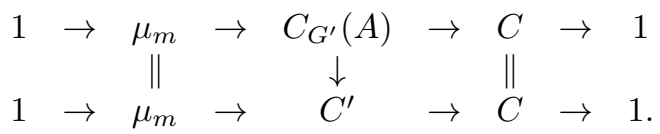

Proof. We choose a local subring $\mathcal{O}^{\prime}$ of $\mathcal{K}$ obtained by adding a primitive $\left|O_{p^{\prime}}(G)\right|$-th root $\xi$ of unity to the ring of rational integers $\mathbb{Z}$ and localizing $\mathbb{Z}[\xi]$ at a maximal ideal containing $q$; then $\left|O_{p^{\prime}}(G)\right|$ is invertible in $\mathcal{O}^{\prime}$ and the residue field $k^{\prime}$ of $\mathcal{O}^{\prime}$ is equal to $\mathbb{F}_{q}(\xi)$ and has characteristic $q$. Let $\alpha$ be the unique character of $\mathcal{O} O_{p^{\prime}}(G) f$. Then for any $a \in \mathcal{O} O_{p^{\prime}}(G) f$, by the Fourier inversion formula, we have

$$
a=\frac{\alpha(1)}{\left|O_{p^{\prime}}(G)\right|} \sum_{z \in O_{p^{\prime}}(G)} \alpha\left(a z^{-1}\right) z .
$$

Whenever $a$ takes $f$ or $s_{x}$ for $x \in C, a z^{-1}$ has finite order dividing $m$. Therefore $\alpha\left(a z^{-1}\right) \in \mathbb{Z}[\xi], f \in\left(\mathcal{O}^{\prime} O_{p^{\prime}}(G)\right)^{A}$ and $s_{x} \in\left(\mathcal{O}^{\prime} O_{p^{\prime}}(G)\right)^{A}$ for any $x \in C$. Furthermore $s_{x} \in\left(\mathcal{O}^{\prime} O_{p^{\prime}}(G) f\right)^{A}$ for any $x \in C$ since $s_{x} f=s_{x}$. Similarly $g \in \mathcal{O}^{\prime} \mathcal{O}_{p^{\prime}}(C)$ and $t_{y} \in \mathcal{O}^{\prime} \mathcal{O}_{p^{\prime}}(C) g$ for any $y \in C$.

We denote by $\bar{g}$ the image of $g$ in $k^{\prime} \mathcal{O}_{p^{\prime}}(C)$. Since $b r_{A}\left(s_{x}\right)$ and $b r_{A}\left(t_{x}\right)$ both act on $k^{\prime} \mathcal{O}_{p^{\prime}}(C) \bar{g}$ as $x$ for any $x \in C$, there is a unique $\nu_{x} \in k^{\prime *}$ such that $b r_{A}\left(s_{x}\right)=$ $\nu_{x} b r_{A}\left(t_{x}\right)$. Moreover since $b r_{A}\left(s_{x}\right)$ and $b r_{A}\left(t_{x}\right)$ both have order dividing $m, \nu_{x}$ has finite order dividing $m$. Therefore we can uniquely lift $\nu_{x}$ to an invertible element of $\mathcal{O}^{\prime}$ with the same order as $\nu_{x}$; we still denote this invertible element by $\nu_{x}$. Then by the proof of [5, 4.5.8], we can prove that there is an isomorphism of $\mathcal{O}^{*}$-groups

$$
\tilde{C} \cong \hat{C}
$$

which maps $\left(x, t_{x}\right)$ onto $\left(x, \nu_{x} s_{x}\right)$ for any $x \in C$ and $(x, x g)$ onto $(x, x f)$ for any $x \in$ $O_{p^{\prime}}(C)$. In particular, the isomorphism (17.2) induces an isomorphism $C_{G^{\prime}}(A) \cong$ $C^{\prime}$. The latter preserves $O_{p^{\prime}}(C)$ element-wise and fulfilling (17.1), but unfortunately it does not preserve $R$ element-wise. So we have to adjust the isomorphism (17.2).

Since $R \subset C_{G^{\prime}}(A)$ and $R \subset C^{\prime}$ (refer to (12.1) and paragraph 14), without loss of generality, we take $t_{u}$ to be $\varsigma(u)$ and $s_{u}$ to be $\sigma(u)$ for any $u \in R$. Then the isomorphism $\tilde{C} \cong \hat{C}$ above implies that for any $u, v \in R, \nu_{u} \nu_{v}=\nu_{u v}$. This shows that the function $\nu: R \rightarrow \mathbb{Z}[\xi], u \mapsto \nu_{u}$ is a linear character of $R$. We claim that $\nu$ is $C$-stable. By the uniqueness of $\sigma$ and $\varsigma$, we have $x \sigma(u) x^{-1}=\sigma\left(x u x^{-1}\right)$ and $x \varsigma(u) x^{-1}=\varsigma\left(x u x^{-1}\right)$ for any $x \in C$ and $u \in R$ such that $x u x^{-1} \in R$. Then we have

$\nu\left(x u x^{-1}\right)=b r_{A}\left(\sigma\left(x u x^{-1}\right)\right) b r_{A}\left(\varsigma\left(x u x^{-1}\right)\right)=b r_{A}\left(x \sigma(u) x^{-1}\right) b r_{A}\left(x \varsigma(u) x^{-1}\right)=\nu(u)$.

The claim is proved. 
Notice that $R$ is a Sylow $p$-subgroup of $C$. Then by [5, Prop. 2.6], $\nu$ can be extended to a linear character of $C$ and we still denote this character by $\nu$. By using $\nu$, we define a map

$$
\tilde{C} \rightarrow \hat{C},\left(x, t_{x}\right) \mapsto \nu\left(x^{-1}\right) \nu_{x}\left(x, s_{x}\right) .
$$

Then it is easily checked that this map is an isomorphism of $\mathcal{O}^{*}$-groups and its inverse induces the desired isomorphism $\Phi: C_{G^{\prime}}(A) \cong C^{\prime}$ in the lemma.

18. We define a function $\alpha^{\prime}$ on $G^{\prime}$ by setting

$$
\alpha^{\prime}(x, s)=\alpha(s)
$$

for any $(x, s) \in G^{\prime}$. Then $\alpha^{\prime}$ is an irreducible character of $G^{\prime}$ extending $\alpha$ through the inclusion $O_{p^{\prime}}(G) \hookrightarrow G^{\prime}$. Since $\mathcal{O} O_{p^{\prime}}(G) f$ is a full matrix algebra over $\mathcal{O}$, by the Skolem-Noether theorem, for any $(x, s) \in G^{\prime}$ and $a \in A, a(s)$ is equal to some conjugate of $s$ in $\mathcal{O} O_{p^{\prime}}(G) f$. Therefore $\alpha^{\prime}(a(x, s))=\alpha^{\prime}(x, s)$ and $\alpha^{\prime}$ is $A$-stable. Similarly a function $\beta^{\prime}$ on $C^{\prime}$ defined by

$$
\beta^{\prime}(y, t)=\beta(t)
$$

for any $(y, t) \in C^{\prime}$ is also an irreducible character of $C^{\prime}$, which extends $\beta$ through the inclusion $O_{p^{\prime}}(C) \hookrightarrow C^{\prime}$.

Lemma 19. Keep the notation and hypotheses as above. Then there is a linear character $\gamma^{\prime}: C^{\prime} \rightarrow \mathcal{O}$ such that the following hold:

19.1. $\mu_{m} \subset \operatorname{Ker}\left(\gamma^{\prime}\right)$ and $\operatorname{Im}\left(\gamma^{\prime}\right) \subset \mu_{m}$.

19.2. The map $\Theta: C_{G^{\prime}}(A) \rightarrow C^{\prime},(t, y) \mapsto \gamma^{\prime}(\Phi(t, y)) \Phi(t, y)$ is an isomorphism preserving $O_{p^{\prime}}(C)$ elementwise.

19.3. $\pi\left(G^{\prime}, A\right)\left(\alpha^{\prime}\right) \circ \Theta^{-1}=\beta^{\prime}$.

Proof. By [5, Th. 2.3], $\pi\left(G^{\prime}, A\right)\left(\alpha^{\prime}\right) \circ \Phi^{-1}$ is an irreducible character of $C^{\prime}$ and is an extension of $\beta$. Therefore there is a linear character $\gamma^{\prime}: C^{\prime} \rightarrow \mathcal{O}$ of $C^{\prime}$ such that $\pi\left(G^{\prime}, A\right)\left(\alpha^{\prime}\right) \circ \Phi^{-1}=\gamma^{\prime} \beta^{\prime}$ and $O_{p^{\prime}}(C) \subset \operatorname{Ker}(\gamma)$. Take $\xi \in \mu_{m}$. Since

$$
\left(\pi\left(G^{\prime}, A\right)\left(\alpha^{\prime}\right) \circ \Phi^{-1}\right)(1, \xi g)=\xi\left(\pi\left(G^{\prime}, A\right)\left(\beta^{\prime}\right) \circ \Phi^{-1}\right)(1, g)=\xi \beta(1)=\beta^{\prime}(\xi g, 1),
$$

$\gamma^{\prime}(\xi)=1$ and $\mu_{m} \subset \operatorname{Ker}\left(\gamma^{\prime}\right)$. The inclusion $\operatorname{Im}\left(\gamma^{\prime}\right) \subset \mu_{m}$ is obvious since the exponent of $C^{\prime}$ divides $m$. So up to now, 19.1 is proved. The latter two can be trivially verified.

Lemma 20. Keep the notation and hypotheses as above. Then $C_{\bar{G}}(A)=$ $C_{G}(A) O_{p^{\prime}}(G) / O_{p^{\prime}}(G)$ and we have an isomorphism

$$
C_{\bar{G}}(A) \cong \bar{C}, x O_{p^{\prime}}(G) \mapsto x O_{p^{\prime}}(C) .
$$

Proof. Obviously $C_{G}(A) O_{p^{\prime}}(G) / O_{p^{\prime}}(G) \subset C_{\bar{G}}(A)$. Let $x O_{p^{\prime}}(G) \in C_{\bar{G}}(A)$. We consider the $O_{p^{\prime}}(G) \rtimes A$ on $O_{p^{\prime}}(G) x O_{p^{\prime}}(G)$ defined by the left multiplication of $O_{p^{\prime}}(G)$ on $x O_{p^{\prime}}(G)$ and the obvious action of $A$ on $x O_{p^{\prime}}(G)$ induced by the action of $A$ on $G$. Since $A$ and $O_{p^{\prime}}(G)$ have co-prime order and $O_{p^{\prime}}(G)$ acts regularly on $x O_{p^{\prime}}(G)$, by [3, Lemma 13.8 and Cor. 13.9], the set of all $A$-stable elements in $x O_{p^{\prime}}(G)$ is non-empty and $C_{O_{p^{\prime}}(G)}(A)$ acts transitively on it. This shows that $C_{\bar{G}}(A) \subset C_{G}(A) O_{p^{\prime}}(G) / O_{p^{\prime}}(G)$. Consequently $C_{G}(A) O_{p^{\prime}}(G) / O_{p^{\prime}}(G)=C_{\bar{G}}(A)$. By this equality and the assumption that $C_{G}(A) \cap O_{p^{\prime}}(G)=O_{p^{\prime}}(C)$, it is trivial to see that (20.1) is an isomorphism. 
Below, we always identify $C_{\bar{G}}(A)$ with $\bar{C}$ through the isomorphism (20.1) and thus $\bar{C}$ is a subgroup of $\bar{G}$.

Lemma 21. Keep the notation and hypotheses as above. Then we have $C_{\hat{\bar{G}}}(A)=\hat{\bar{C}}$. Proof. Set $\bar{G}^{\prime}=G^{\prime} / O_{p^{\prime}}(G)$. By Lemma $11, \bar{G}^{\prime}$ is a subgroup of $\hat{\bar{G}}$ such that $\mathcal{O}^{*} \bar{G}^{\prime}=\hat{\bar{G}}$. Then replacing $\hat{G}$ by $\hat{\bar{G}}$ and $G^{\prime}$ by $\bar{G}^{\prime}$ in the proof of Lemma 16 , we can prove this lemma.

Lemma 22. Keep the notation and hypotheses as above and denote by

$$
\Gamma: \hat{C} \cong \tilde{C}
$$

the isomorphism induced by $\Theta$ (see Lemma 19). Then $\Gamma$ induces an isomorphism of $\mathcal{O}^{*}$-groups

$$
\bar{\Gamma}: \hat{\bar{C}} \cong \tilde{\bar{C}} .
$$

Proof. Since $\Gamma$ maps $\hat{C} \cap O_{p^{\prime}}(G)$ onto $O_{p^{\prime}}(C)$, it induces an isomorphism of $\mathcal{O}^{*}$ groups

$$
\bar{\Gamma}: \hat{C} O_{p^{\prime}}(G) / O_{p^{\prime}}(G) \cong \hat{C} / \hat{C} \cap O_{p^{\prime}}(G) \cong \tilde{C} / O_{p^{\prime}}(C)=\tilde{\bar{C}}
$$

By Lemma 16, $\hat{C}=C_{\hat{G}}(A)$; therefore $\hat{C} O_{p^{\prime}}(G) / O_{p^{\prime}}(G)=C_{\hat{G}}(A) O_{p^{\prime}}(G) / O_{p^{\prime}}(G) \subset$ $C_{\hat{G}}(A)$. On the other hand, it follows from Lemmas 20 and 21 that $\hat{C} O_{p^{\prime}}(G) / O_{p^{\prime}}(G)$ and $C_{\hat{\bar{G}}}(A)$ both are $\mathcal{O}^{*}$-groups with the $\mathcal{O}^{*}$-quotient $\bar{C}$. Therefore the above inclusion $C_{\hat{G}}(A) O_{p^{\prime}}(G) / O_{p^{\prime}}(G) \subset C_{\hat{G}}(A)$ should be an equality. Then $\bar{\Gamma}$ is the desired isomorphism.

Obviously the group isomorphisms $\Gamma$ and $\bar{\Gamma}$ induce isomorphisms

$$
\mathcal{O}_{*} \hat{C} \cong \mathcal{O}_{*} \tilde{C} \text { and } \mathcal{O}_{*} \hat{\bar{C}}^{\circ} \cong \mathcal{O}_{*} \tilde{\bar{C}}^{\circ}
$$

We still denote these isomorphisms by $\Gamma$ and $\bar{\Gamma}$ respectively.

Proposition 23. Keep the notation and hypotheses as above. Then there is a bijection $\pi\left(\hat{\bar{G}}^{\circ}, A\right): \operatorname{Irr}\left(\hat{\bar{G}}^{\circ}, \bar{b}\right) \rightarrow \operatorname{Irr}\left(\tilde{\bar{C}}^{\circ}, \bar{c}\right)$ such that

23.1. For any $\bar{\chi} \in \operatorname{Irr}\left(\hat{\bar{G}}^{\circ}, \bar{b}\right), \pi\left(\hat{\bar{G}}^{\circ}, A\right)(\bar{\chi}) \circ \bar{\Gamma}^{-1}$ is the unique irreducible constituent of the restriction of $\bar{\chi}$ to $\mathcal{K} \otimes_{\mathcal{O}} \mathcal{O}_{*} \hat{\bar{C}}^{\circ}$, which occurs with a multiplicity prime to $q$.

23.2. If $\chi \in \operatorname{Irr}(G, b)$ corresponds to $\bar{\chi} \in \operatorname{Irr}(\bar{b})$ through the bijection (13.3), then $\pi(G, A)(\chi)$ corresponds to $\pi\left(\hat{\bar{G}}^{\circ}, A\right)(\bar{\chi})$ through the bijection (15.3).

Proof. In this proof, we will identify $\hat{C}$ with $\tilde{C}$ through $\Gamma$ and $\hat{\bar{C}}$ with $\tilde{\bar{C}}$ through $\bar{\Gamma}$.

By our hypothesis, the map $\pi(G, A, b): \operatorname{Irr}(G, b) \rightarrow \operatorname{Irr}(C, c), \chi \mapsto \pi(G, A)(\chi)$ is a bijection. We denote by $\pi\left(\hat{\bar{G}}^{\circ}, A\right)$ the composition of the inverse of the bijection (13.3), $\pi(G, A, b)$ and the bijection (15.3). Take $\chi \in \operatorname{Irr}(G, b)$ and set $\psi=\pi(G, A)(\chi)$; then $\pi\left(\hat{\bar{G}}^{\circ}, A\right)(\bar{\chi})=\bar{\psi}$. Then in order to prove the proposition above, it suffices to show that $\bar{\psi}$ is the unique irreducible constituent of the restriction of $\bar{\chi}$ to $\mathcal{K} \otimes_{\mathcal{O}} \mathcal{O}_{*} \tilde{\bar{C}}^{\circ}$, which occurs with a multiplicity prime to $q$.

We inflate $\chi$ to $\chi^{\prime}$ through the canonical surjective homomorphism $G^{\prime} \rightarrow G$ and $\psi$ to $\psi^{\prime}$ through the canonical surjective homomorphism $C^{\prime} \rightarrow C$. Since $\pi(G, A)(\chi)=$ $\psi, \pi\left(G^{\prime}, A\right)\left(\chi^{\prime}\right)=\psi^{\prime}$. We define a function $\bar{\chi}^{\prime}$ on $G^{\prime}$ by setting $\bar{\chi}^{\prime}(x, s)=\bar{\chi}(\overline{(x, s)})$ for any $(x, s) \in G^{\prime}$. Then $\bar{\chi}^{\prime}$ is an irreducible character of $G^{\prime}$ and it follows from the 
isomorphism (13.3) that $\chi^{\prime}(x, s)=\alpha^{\prime}(x, s) \bar{\chi}^{\prime}(x, s)$ for any $(x, s) \in G^{\prime}$. Moreover since $\chi^{\prime}$ and $\alpha^{\prime}$ are $A$-stable, so is $\bar{\chi}^{\prime}$. Similarly the function $\bar{\psi}^{\prime}$ on $C^{\prime}$ by defining $\bar{\psi}^{\prime}(y, t)=\bar{\psi}(\overline{(y, t)})$ for any $(y, t) \in C^{\prime}$ is also an irreducible character of $C^{\prime}$ and fulfills the equality $\psi^{\prime}(y, t)=\beta^{\prime}(y, t) \bar{\psi}^{\prime}(y, t)$ for any $(y, t) \in C^{\prime}$.

We decompose the restriction $\operatorname{Res}_{C^{\prime}}^{G^{\prime}}\left(\alpha^{\prime}\right)$ as the sum $\sum_{1 \leq i \leq n} \alpha_{i}^{\prime}$ of irreducible characters of $C^{\prime}$ and the restriction $\operatorname{Res}_{C^{\prime}}^{G^{\prime}}\left(\bar{\chi}^{\prime}\right)$ as the sum $\sum_{1 \leq j \leq m} \bar{\chi}_{j}^{\prime}$. Then $\operatorname{Res}_{C^{\prime}}^{G^{\prime}}\left(\chi^{\prime}\right)=\sum_{1 \leq i \leq n} \sum_{1 \leq j \leq m} \alpha_{i}^{\prime} \bar{\chi}_{j}^{\prime}$. Now we claim that if $\psi^{\prime}$ is an irreducible constituent of some $\alpha_{i}^{\prime} \bar{\chi}_{j}^{\prime}$, then

$$
\alpha_{i}^{\prime}=\beta^{\prime} \quad \text { and } \quad \bar{\chi}_{j}^{\prime}=\bar{\psi}^{\prime} .
$$

Since $\pi\left(G^{\prime}, A\right)\left(\chi^{\prime}\right)=\psi^{\prime}$ and $\pi\left(G^{\prime}, A\right)\left(\alpha^{\prime}\right)=\beta^{\prime}$, this implies that the multiplicity of $\bar{\psi}^{\prime}$ in the restriction $\operatorname{Res}_{C^{\prime}}^{G^{\prime}}\left(\bar{\chi}^{\prime}\right)$ is prime to $q$. In particular, the multiplicity of $\bar{\psi}$ in the restriction of $\bar{\chi}$ to $\mathcal{K} \otimes_{\mathcal{O}} \mathcal{O}_{*} \tilde{\bar{C}}^{\circ}$ is prime to $q$; the uniqueness of $\bar{\psi}$ follows from [2, Th. 6.13].

Suppose $\alpha_{i}^{\prime} \neq \beta^{\prime}$. Since $g$ is a central idempotent of $\mathcal{O} C^{\prime}$ and $\psi^{\prime}$ is a character of $C^{\prime}$ provided by some irreducible $\mathcal{K} C^{\prime} g$-module (refer to the isomorphisms (15.1) and $(15.2)), \alpha_{i}^{\prime}(g) \bar{\chi}_{j}^{\prime}(g) \neq 0$ and thus $\alpha_{i}^{\prime}(g) \neq 0$. But since $\alpha_{i}^{\prime}$ is irreducible, $\alpha_{i}^{\prime}$ is a character of some irreducible $\mathcal{K} C^{\prime} g$-module and the restriction $\operatorname{Res}_{O_{p^{\prime}}(C)}^{C^{\prime}}\left(\alpha_{i}^{\prime}\right)$ is some multiple of $\beta$. On the other hand, since $\pi\left(G^{\prime}, A\right)\left(\alpha^{\prime}\right)=\beta^{\prime}$, there are $\alpha_{i_{1}}^{\prime}, \alpha_{i_{2}}^{\prime}$, $\cdots, \alpha_{i_{l}}^{\prime}$ among $\alpha_{1}^{\prime}, \alpha_{2}^{\prime}, \ldots, \alpha_{n}^{\prime}$ such that $\alpha_{i_{1}}^{\prime}=\alpha_{i_{2}}^{\prime}=\cdots=\alpha_{i_{l}}^{\prime}=\beta^{\prime}$, where $l$ is the multiplicity of $\beta^{\prime}$ in the restriction of $\alpha^{\prime}$ to $C^{\prime}$. Therefore since

$$
\operatorname{Res}_{O_{p^{\prime}}(C)}^{C^{\prime}}\left(\operatorname{Res}_{C^{\prime}}^{G^{\prime}}\left(\alpha^{\prime}\right)\right)=\operatorname{Res}_{O_{p^{\prime}}(C)}^{O_{p^{\prime}}(G)}\left(\operatorname{Res}_{O_{p^{\prime}}(G)}^{G^{\prime}}\left(\alpha^{\prime}\right)\right)=\operatorname{Res}_{O_{p^{\prime}}(C)}^{O_{p^{\prime}}(G)}(\alpha),
$$

the multiplicity of $\beta$ in $\operatorname{Res}_{O_{p^{\prime}}(C)}^{O_{p^{\prime}}(G)}(\alpha)$ is greater than the sum of $l$ with the multiplicity of $\beta$ in $\operatorname{Res}_{O_{p^{\prime}}(C)}^{C^{\prime}}\left(\alpha_{i}^{\prime}\right)$ and thus strictly exceeds $l$. But the multiplicity of $\beta$ in $\operatorname{Res}_{O_{p^{\prime}}(C)}^{O_{p^{\prime}}(G)}(\alpha)$ is $l$. So a contradiction is produced and $\alpha_{i}^{\prime}=\beta^{\prime}$.

Note that $\bar{\chi}_{j}^{\prime}$ is an irreducible constituent of $\operatorname{Res}_{C^{\prime}}^{G^{\prime}}\left(\bar{\chi}^{\prime}\right)$ and that $\bar{\chi}$ is a character of an irreducible $\mathcal{K} \otimes_{\mathcal{O}} \mathcal{O}_{*} \hat{\bar{G}}^{\circ}$-module. Therefore there is a character $\bar{\chi}_{j}$ of some irreducible $\mathcal{K} \otimes_{\mathcal{O}} \mathcal{O}_{*} \hat{\bar{C}}^{\circ}$-module such that for any $(x, s) \in C^{\prime}, \bar{\chi}_{j}^{\prime}(x, s)=\bar{\chi}_{j}(x, s)$. Then by the isomorphism (15.1), $\alpha_{i}^{\prime} \bar{\chi}_{j}^{\prime}$ is an irreducible character of $G^{\prime}$. So $\psi^{\prime}=$ $\alpha_{i}^{\prime} \bar{\chi}_{j}^{\prime}$ and $\bar{\chi}_{j}^{\prime}=\bar{\psi}^{\prime}$.

24. A proof of Theorem 2. In order to avoid unnecessary repetition, we continue to keep all the notation from paragraph 5 to paragraph 23. First we consider the case that the block idempotent $f$ of $\mathcal{O} O_{p^{\prime}}(G)$ (see paragraph 2.1) is not stabilized by $G$. Then we have isomorphisms (see (6.2) and (8.1))

$$
\operatorname{Ind}_{K}^{G}(\mathcal{O} K d) \cong \mathcal{O} G b \quad \text { and } \quad \operatorname{Ind}_{C_{K}(A)}^{C_{G}(A)}\left(\mathcal{O} C_{K}(A) e\right) \cong \mathcal{O} C_{G}(A) c .
$$

Suppose that the $\mathcal{O}\left(C_{K}(A) \times K\right)$-module $N$ induces a Morita equivalence between $\mathcal{O} K d$ and $\mathcal{O C}_{K}(A) e$, which induces the Glauberman correspondence of characters from $\operatorname{Irr}(K, d)$ to $\operatorname{Irr}\left(C_{K}(A), e\right)$. Then it is trivial to see that the $\mathcal{O}\left(C_{G}(A) \times G\right)$ module $M=\mathcal{O}\left(C_{G}(A) \times G\right) \otimes_{\mathcal{O}\left(C_{K}(A) \times K\right)} N$ induces a Morita equivalence between $\mathcal{O} G b$ and $\mathcal{O} C c$. Take $\chi \in \operatorname{Irr}(K, d)$. Let $W$ be a $\mathcal{K} K d$-module providing the 
character $\chi$. Since

$$
\begin{aligned}
\left(\mathcal{K} \otimes_{\mathcal{O}} M\right) \otimes_{\mathcal{K} G}\left(\mathcal{K} G \otimes_{\mathcal{K} K} W\right) \cong & \left(\mathcal{K} C_{G}(A) \otimes_{\mathcal{K} C_{K}(A)}\left(\mathcal{K} \otimes_{\mathcal{O}} N\right) \otimes_{\mathcal{K} K} \mathcal{K} G\right) \\
& \otimes_{\mathcal{K} G}\left(\mathcal{K} G \otimes_{\mathcal{K} K} W\right) \\
& \cong \mathcal{K}_{G}(A) \otimes_{\mathcal{K} C_{K}(A)}\left(\left(\mathcal{K} \otimes_{\mathcal{O}} N\right) \otimes_{\mathcal{K} K} W\right)
\end{aligned}
$$

and the character of $\left(\mathcal{K} \otimes_{\mathcal{O}} N\right) \otimes_{\mathcal{K} K} W$ is $\pi(K, A)(\chi)$, then by [5, Th. 2.3], the Morita equivalence induced by $M$ induces the Glauberman correspondence of characters from $\operatorname{Irr}(G, b)$ to $\operatorname{Irr}(C, c)$. Here we note that $N$ and $M$ have common vertexes and source modules.

Therefore in order to prove Theorem 2, we can assume without loss of generality that $f$ is stabilized by $G$. Then we have the algebra isomorphisms (see (13.2) and $(15.2))$

$$
\mathcal{O} G b \cong \mathcal{O}_{p^{\prime}}(G) f \otimes_{\mathcal{O}} \mathcal{O}_{*} \hat{\bar{G}}^{\circ} \bar{b} \quad \text { and } \quad \mathcal{O} C c \cong \mathcal{O}_{p^{\prime}}(C) g \otimes_{\mathcal{O}} \mathcal{O}_{*} \tilde{\bar{C}}^{\circ} \bar{c},
$$

group isomorphisms (see Lemma 22)

$$
\Gamma: \hat{C} \cong \tilde{C} \quad \text { and } \quad \bar{\Gamma}: \hat{\bar{C}} \cong \tilde{\bar{C}}
$$

and isomorphisms of algebras

$$
\Gamma: \mathcal{O}_{*} \hat{C} \cong \mathcal{O}_{*} \tilde{C} \quad \text { and } \quad \bar{\Gamma}: \mathcal{O}_{*} \hat{\bar{C}}^{\circ} \cong \mathcal{O}_{*} \tilde{\bar{C}}^{\circ} .
$$

Let $i$ be a primitive idempotent of $\mathcal{O} O_{p^{\prime}}(G) f$ and $j$ be a primitive idempotent of $\mathcal{O} O_{p^{\prime}}(C) g$. It is well known that the $\mathcal{O}_{*} \hat{\bar{G}}^{\circ} \bar{b} \otimes_{\mathcal{O}} \mathcal{O} G b$-module $i \mathcal{O} O_{p^{\prime}}(G) \otimes_{\mathcal{O}} \mathcal{O}_{*} \hat{\bar{G}}^{\circ} \bar{b}$ induces a Morita equivalence between $\mathcal{O} G b$ and $\mathcal{O}_{*} \hat{\bar{G}}^{\circ} \bar{b}$, which induces the bijection (13.3) of characters, and that the $\mathcal{O} C c \otimes_{\mathcal{O}} \mathcal{O}_{*} \tilde{\bar{C}}^{\circ} \bar{c}$-module $\mathcal{O}_{p^{\prime}}(C) j \otimes_{\mathcal{O}} \mathcal{O}_{*} \tilde{\bar{C}}^{\circ} \bar{c}$ induces a Morita equivalence between $\mathcal{O}_{*} \tilde{\bar{C}}^{\circ} \bar{c}$ and $\mathcal{O} C c$, which induces the inverse of the bijection (15.3) of characters. Moreover since we assume $G=O_{p^{\prime}}(G) C_{G}(A)$, $\hat{\bar{C}}=\hat{\bar{G}}$ and then it follows from Proposition 23 that $\bar{\Gamma}$ maps $\mathcal{O}_{*} \hat{\bar{G}}^{\circ} \bar{b}$ onto $\mathcal{O}_{*} \tilde{\bar{C}}^{\circ} \bar{c}$ isomorphically; in this case, it is clear that the $\mathcal{O}_{*} \tilde{\bar{C}}^{\circ} \otimes_{\mathcal{O}} \mathcal{O}_{*} \hat{\bar{G}}^{\circ}$-module $\bar{\Gamma}^{-1}\left(\mathcal{O}_{*} \hat{\bar{G}}^{\circ} \bar{b}\right)$, which is defined by the equality $((1 \otimes x) \otimes(1 \otimes y)) \cdot a=\bar{\Gamma}^{-1}(1 \otimes x) a\left(1 \otimes y^{-1}\right)$ for any $x \in \tilde{\bar{C}}, y \in \hat{\bar{G}}$ and $a \in \mathcal{O}_{*} \hat{\bar{G}}{ }^{\circ} \bar{b}$, induces a Morita equivalence between $\mathcal{O}_{*} \hat{\bar{G}}^{\circ} \bar{b}$ and $\mathcal{O}_{*} \tilde{\bar{C}}^{\circ} \bar{c}$. Then we can easily see that the $\mathcal{O}(C \times G)$-module

$$
\begin{aligned}
\left(\mathcal{O} O_{p^{\prime}}(C) j \otimes_{\mathcal{O}} \mathcal{O}_{*} \tilde{\bar{C}}^{\circ} \bar{c}\right) & \otimes_{\mathcal{O}_{*} \tilde{\bar{C}}^{\circ}}\left(i \mathcal{O} O_{p^{\prime}}(G) \otimes_{\mathcal{O}} \bar{\Gamma}^{-1}\left(\mathcal{O}_{*} \hat{\bar{G}}^{\circ} \bar{b}\right)\right) \\
& \cong \mathcal{O} O_{p^{\prime}}(C) j \otimes_{\mathcal{O}} \bar{\Gamma}^{-1}\left(\mathcal{O}_{*} \hat{\bar{G}}^{\circ} \bar{b}\right) \otimes_{\mathcal{O}} i \mathcal{O} O_{p^{\prime}}(G)
\end{aligned}
$$

induces a Morita equivalence between $\mathcal{O} G b$ and $\mathcal{O} C c$ (note that $\mathcal{O}(C \times G) \cong \mathcal{O} C \otimes_{\mathcal{O}}$ $\mathcal{O} G$ ); moreover by Proposition 23, the bijection of characters induced by this Morita equivalence coincides with the Glauberman correspondence of characters between $\operatorname{Irr}(G, b)$ and $\operatorname{Irr}(C, c)$.

Finally, employing the fifth paragraph in the proof of 1.1 in the reduced case in [5], we can prove that $\mathcal{O} O_{p^{\prime}}(C) j \otimes_{\mathcal{O}} \bar{\Gamma}^{-1}\left(\mathcal{O}_{*} \hat{\bar{G}}^{\circ} \bar{b}\right) \otimes_{\mathcal{O}} i \mathcal{O} O_{p^{\prime}}(G)$ has $\Delta(P)$ as a vertex. 


\section{ACKNOWLEDGEMEnts}

During the preparation of this paper, the author was supported by NSFC (Grant No. 10501016), the Key Project of Chinese Ministry of Education, and the Alexander von Humboldt Foundation of Germany; he is very thankful for this financial support. Thanks are also given to the referee for a careful reading of this paper.

\section{REFERENCES}

[1] M. Broué, Isométries parfaites, types de blocs, catégories dérivées. Astérisque No. 181-182 (1990), 61-92. MR 1051243 (91i:20006)

[2] E. Dade, A new approach to Glauberman's correspondence. J. Algebra 270 (2003), no. 2, 583-628. MR2019631(2004j:20010)

[3] Y. Fan and L. Puig, On blocks with nilpotent coefficient extensions. Algebr. Represent. Theory 1 (1998), no. 1, 27-73. MR.1654602 (2000e:20019a)

[4] G. Glauberman, Correspondences of characters for relatively prime operator groups. Can. J. Math. 20 (1968), 1465-1488. MR0232866 (38:1189)

[5] M. E. Harris and M. Linckelmann, On the Glauberman and Watanabe correspondences for blocks of finite $p$-solvable groups. Trans. Amer. Math. Soc. 354 (2002), no. 9, 3435-3453. MR1911507 (2003c:20008)

[6] I. M. Isaacs, Character theory of finite groups. Academic Press, New York, 1976. MR0460423 $(57: 417)$

[7] L. Puig, Nilpotent blocks and their source algebras. Invent. Math. 93 (1988), 77-116. MR.943924 (89e:20023)

[8] L. Puig, Notes on $\mathcal{O}^{*}$-groups, preprint, 1998.

[9] A. Watanabe, The Glauberman character correspondence and perfect isometries for blocks of finite groups. J. Algebra 216 (1999), 548-565. MR.1692989 (2000f:20015)

[10] J. Thévenaz, $G$-algebras and modular representation theory. Oxford Mathematical Monographs, Oxford Science Publications, The Clarendon Press, Oxford University Press, New York, 1995. MR1365077 (96j:20017)

Department of Mathematics and Statistics, Central China Normal University, Wuhan, 430079, People's Republic of China

E-mail address: zhouyy74@163.com 